\title{
Early Fasciotomy in Patients with Extremity Vascular Injury is Associated with Decreased Risk of Adverse Limb Outcomes: A Review of the National Trauma Data Bank
}

\author{
Alik Farber, Tze-Woei Tan ${ }^{1}$, Naomi M Hamburg ${ }^{2}$, Jeffrey A Kalish ${ }^{1}$, Fernando Joglar ${ }^{1}$, \\ Timna Onigman ${ }^{1}$, Denis Rybin ${ }^{3}$, Gheorghe Doros ${ }^{3}$, and Robert T Eberhardt ${ }^{2}$ \\ ${ }^{1}$ Section of Vascular and Endovascular Surgery, Boston University Medical Center, Boston, MA \\ ${ }^{2}$ Section of Cardiovascular Medicine, Boston University Medical Center, Boston, MA \\ ${ }^{3}$ Department of Public Health, Boston University, Boston, MA
}

\section{Abstract}

\begin{abstract}
Introduction and Objectives-Lower Extremity (LE) arterial trauma and its treatment may lead to extremity compartment syndrome (ECS). In that setting, the decision to perform fasciotomies is multifactoral and is not well delineated. We evaluated the outcomes of patients with surgically treated LE arterial injury who underwent early or delayed fasciotomies.
\end{abstract}

\begin{abstract}
Methods-The National Trauma Data Bank (NTDB) was retrospectively reviewed for patients who had LE arterial trauma and underwent both open vascular repair and fasciotomies. Exclusion criteria were additional non-LE vascular trauma, head or spinal cord injuries, crush injuries, burn injuries, and declaration of death on arrival. Patients were divided into those who had fasciotomies performed within 8 hours (Early Group) or $>8$ hours after open vascular repair (Late Group). Comparative analyses of demographics, injury characteristics, complications, and outcomes were performed.
\end{abstract}

Results-Of the 1,469 patient admissions with lower extremity arterial trauma that met inclusion criteria there were 612 patients $(41.7 \%)$ who underwent fasciotomies. There were 543 and 69 patients in the Early and Late Fasciotomy Groups, respectively. There was no significant difference in age, injury severity, mechanism of injury, associated injuries, and type of vascular repair between the groups. A higher rate of iliac artery injury was observed in the Late Fasciotomy Group (23.2\% vs. 5.9\%, P<.001). Patients in the Early Fasciotomy Group had lower amputation rate $(8.5 \%$ vs. $24.6 \%, \mathrm{P}<.001)$, lower infection rate $(6.6 \%$ vs. $14.5 \%, \mathrm{P}=.028)$ and shorted total hospital stay (18.5 \pm 20.7 days vs. $24.2 \pm 14.7$ days, $\mathrm{P}=.007)$ than those in the Late Fasciotomy Group. On multivariable analysis, early fasciotomy was associated with a 4-fold lower risk of amputation (Odds Ratio 0.26, 95\% CI 0.14-0.50, p<.0001) and 23\% shorter hospital LOS (Means Ratio $0.77,95 \%$ CI $0.64-0.94, \mathrm{P}=.01$ ).

Conclusion-Early fasciotomy is associated with improved outcomes in patients with lower extremity vascular trauma treated with surgical intervention. Our findings suggest that appropriate implementation of early fasciotomy may reduce amputation rates in extremity arterial injury.

\section{Keywords}

Extremity vascular trauma; arterial trauma; Compartment syndrome; Fascitomy; Early Fasciotomy

Address for correspondence: Alik Farber, MD, Section of Vascular and Endovascular Surgery, Boston University Medical Center, 88 East Newton Street, Collamore Building $5^{\text {th }}$ Floor, Boston, MA 02119-2393, alik.farber@bmc.org, Telephone number: (617) 638-8488, Fax number: (617) 638-8469. 


\section{Introduction}

Compartment syndrome, a clinical condition resulting from pathologically increased tissue pressure in a non-expansile tissue space, may occur in the setting of lower extremity trauma and is associated with significant morbidity and mortality (1-6). Its prompt diagnosis is mostly based on clinical criteria (6-9), although direct compartment pressure measurements have been used in certain clinical scenarios to confirm a suspected diagnosis (7). Once the diagnosis of an extremity compartment syndrome (ECS) is made treatment entails decompressive fasciotomy $(7,8,10)$.

In the setting of trauma, ECS can occur due to hemorrhage occurring as a result of vascular injury or fracture, direct tissue injury caused by crushing blunt trauma, or ischemiareperfusion that can occur during major arterial or venous injury and subsequent repair. The presence of vascular injury more often results in the development of ECS and has been shown to be highly predictive of the need to perform fasciotomy to reduce the risk of limb loss or death (11).

There is an abundance of evidence that treatment of an existing ECS requires urgent and complete fasciotomy and that a delay in treatment results in significant morbidity $(4,8,12,13)$. However, the role of prophylactic fasciotomy in the setting of vascular trauma is not clear. A number of factors such as prolonged ischemia (10), hypotension (10), combined arterial and venous injury $(6,12)$, popliteal vascular injury $(14,15)$, and massive soft tissue injury (10) may predict the development of ECS. Despite knowledge of such risk factors, the decision to perform and timing of a fasciotomy in a patient with vascular injury without established ECS is not standardized, given the variability of patient presentations and surgeon bias. Proponents of prophylactic, or early fasciotomy, suggest that development of ECS and its associated complications can be avoided $(2-4,7,12,14,16)$. Detractors point out that fasciotomies have associated short (16-18) and long-term (19-21) complications and that that their prophylactic use is unnecessary $(15,18,22)$.

We hypothesized that early fasciotomy in the setting of lower extremity arterial vascular trauma are associated with improved outcomes. We thus used the National Trauma Data Bank (NTDB) to compare characteristics and outcomes of patients undergoing early and delayed fasciotomy.

\section{Methods}

We retrospectively reviewed prospectively collected data from $2002-2006$ in the NTDB (Committee on Trauma, American College of Surgeons. NTDB Version 7.2 Chicago, IL, 2007). The American College of Surgeons (ACS) established the NTDB as a public service to be a repository of trauma-related data voluntarily reported by participating trauma centers. The NTDB is the largest repository of data on trauma patients in the United States. Currently, it contains detailed data on over 2.7 million cases from over 900 U.S. trauma centers (23). All data provided by the NTDB is deidentified and subjected to quality screening for consistency and validity.

Our study population consisted of patients older than 18 years who suffered lower extremity arterial injury, underwent open vascular repair, and fasciotomy. We excluded patients with an admission diagnosis of ECS, patients with vascular injuries to other than lower extremity blood vessels, abdominal vascular injury, intracranial or spinal cord injury, crush injury, and burns. Patients were identified using the International Classification of Diseases, Ninth Revision, Clinical Modification codes (ICD-9 CM) for injuries and procedures (Appendix 
1). This study was approved by the Boston University School of Medicine Institutional Review Board.

Patients were divided into two groups based on whether the fasciotomy procedure was performed early or late relative to the vascular repair based on the time code for the 2 procedures. As shown in Figure 1, the distribution of fasciotomy timing was bimodal. In 531 $(87 \%)$ of cases, the time code for vascular repair and fasciotomy was identical and these patients were placed in the early fasciotomy group. In an additional 12 cases, the coded time for the fasciotomy was less than 8 hours after the vascular repair and given the possibility that the vascular repair may have lasted 8 hours these cases were also classified as early. Patients with fasciotomies greater than 18 hours after the vascular repair were classified as late. In less than $10 \%$ of cases more than one fasciotomy was performed. In those instances, the initial fasciotomy was used for classification.

Demographic characteristics abstracted from patient records included age, gender, race, health insurance type, and hospital region. Injury characteristics included location of injured artery, mechanism of injury, presenting systolic blood pressure (SBP), presenting Glasgow Coma Score (GCS), presenting Injury Severity Score (ISS), and presence of associated venous injury, nerve injury or fracture. Type of target vascular procedure performed was recorded as arterial repair, ligation, or placement of a temporary shunt followed by repair or ligation.

The primary outcome measured was in-hospital amputation rate as defined by a below or above knee amputation. Secondary outcomes included in-hospital mortality, complication rates, and total and Intensive Care Unit (ICU) length of stay (LOS) determination. Complications evaluated included wound infection, deep venous thrombosis (DVT) and pulmonary embolism (PE).

Data are presented as mean \pm standard deviation (SD), as medians with interquartile ranges (for LOS), or as counts and percentages. Clinical characteristics were compared between the with early and late fasciotomy groups using the t-test for continuous symmetrically distributed variables, the Mann-Whitney test for continuous asymmetrically distributed variables, and chi-square and Fisher exact tests for categorical values (Fisher's Exact Test). Two sided p-value of .05 was used to determine statistical significance. To estimate the association between the timing of fasciotomy and outcomes, while adjusting for possible confounders, multivariate regression models were used. For the amputation and mortality endpoints we used multivariate logistic regression models while for the length of stay outcomes we used multivariate gamma regression with a Log Link. We used a backward selection procedure with 0.2 level of alpha to keep variables in the model and selected from the following potential confounders: Age, Gender, Injury Site, Presenting ISS, Target Procedure, Injury Type (Blunt/Penetrating), Fracture, Nerve Injury, and Venous Injury.

Statistical significant was considered when the $P$ value was 0.05 . All analyses were performed using SAS 9.2 (SAS Institute Inc., Cary, NC, USA).

\section{Results}

Among the 1,309,311 medical records in the dataset, there were 4,253 patient admissions with lower extremity arterial trauma. After applying the inclusion and exclusion criteria the total sample size was 1,469 patients. There were 612 patients who underwent fasciotomies. Overall patients in the sample were young and predominantly male (Table 1). Both penetrating and blunt trauma were represented and injuries involved each segment of the lower extremity arterial tree (Table 2). There was a high prevalence of associated venous injury. Arterial repair was the most frequent index vascular procedure (Table 2). 
There was no significant difference in mean age, gender, race, insurance type, and hospital region between the Early Fasciotomy $(n=543)$ and the Late Fasciotomy ( $n=69)$ Groups (Table 1). There were a lower proportion of patients with iliac artery injuries in the Early compared to the Late Fasciotomy Group (Table 2). There was no difference in the proportion of blunt and penetrating trauma between the groups. Injury severity, as measured by presenting SBP, GCS, and ISS was likewise similar between the 2 groups. There was no significant difference in concomitant venous injury or fracture rates between the groups. Finally, a similar proportion of patients were treated with arterial repair, ligation, and use of a temporary shunt (Table 2).

On bivariate analysis, there was no difference in hospital mortality between groups. Similarly, DVT and PE rates were similar between the groups. There was, however, a significantly lower wound infection rate in patients undergoing early fasciotomy. Patients in the Early Fasciotomy Group had a significantly lower amputation rate than those in the Late Fasciotomy Group. In addition, patients in the Early Fasciotomy Group had significantly shorter total LOS than patients in the Late Fasciotomy Group. (Table 3).

In the multivariate analysis adjusting for blunt versus penetrating trauma and ISS, Early Fasciotomy was associated with a 4-fold lower risk of amputation (Odds Ratio $0.26,95 \%$ CI $0.14-0.50, \mathrm{P}<.0001)$. Subgroup analysis revealed that the lower risk for amputation was maintained across subgroups defined by type of vessel injured, mechanism of injury, vascular procedure performed, and presence of associated fracture or venous injury (Figure 2). In a multivariable analysis adjusting for sex, injury location, blunt versus penetrating trauma, injury location, ISS, fracture, nerve injury and venous injury; early fasciotomy was associated with a $23 \%$ shorter hospital LOS (Means Ratio 0.77, 95\% CI 0.64-0.94, P=.01).

Given that iliac artery trauma may in itself be associated with higher morbidity and mortality, an additional analysis were performed after exclusion of all iliac artery injuries from the study group. The case number decreased to 511 in the Early Fasciotomy Group and 53 in the Late Fasciotomy Group. The injury sites and mechanism of injuries were similar between both groups and injury severity, as reflected by SBP, GCS and ISS, were similar on presentation. There were no differences between associated facture, nerve and venous injuries. The most common target procedure was vascular repair and vascular interventions were similar between groups. Bivariate analysis revealed major lower limb amputation ( $22.6 \%$ vs. $8.4 \%, \mathrm{P}=.003$ ) to be significantly higher in the Late Fasciotomy Group and total LOS to be significantly shorter in the Early Fasciotomy Group (17.6 \pm 17.1 vs. $24.1 \pm 15.5$ day, $\mathrm{P}=.003)$.

In order to further evaluate the relationship of fasciotomy to adverse outcomes we performed a secondary analysis that included patients who sustained a lower extremity arterial injury had a vascular repair, met inclusion criteria but did not undergo fasciotomy (No Fasciotomy Group, n=857). The No Fasciotomy Group was significantly different from those patients treated with fasciotomies evidenced by a higher proportion of women, lower proportion of popliteal injury, lower prevalence of associated venous injury or fracture, and a higher rate of arterial ligation (data not shown). Furthermore, this group had lower wound infection and amputation rates. In a multivariable model, we compared patients who had early fasciotomies (Early Fasciotomy Group) with patients without an early fasciotomy (Late Fasciotomy and No Fasciotomy Groups). There was no difference in the risk of amputation between the two groups (Odds Ratio 1.10 95\% CI 0.7, 1.72, P=.67)). 


\section{Discussion}

Vascular trauma occurs in 3\% of all civilian and military injuries and the extremities are involved in $27 \%$ to $87 \%$ of cases (12). Surgical management of extremity arterial trauma is complex and involves both the control of hemorrhage and reestablishment of arterial circulation. Although extremity compartment syndrome (ECS) has multiple possible etiologies, in the setting of arterial trauma it is usually caused by hematoma or ischemiareperfusion (10). Development of ECS is associated with significant morbidity and mortality (1-6) and therefore its immediate presence or potential occurrence needs to be considered during management of extremity arterial trauma. There is little disagreement that upon successful diagnosis ECS needs to be expeditiously treated with fasciotomies $(7,8,10)$. In contrast, the optimal timing of fasciotomy to prevent impending ECS is less clear.

In the present study, we evaluated the effect of fasciotomy timing on outcomes in patients with lower extremity arterial injury in a large national trauma sample. Patients with the diagnosis of compartment syndrome on presentation were excluded from the study. Although the in-hospital mortality rate were similar, we found that among patients with arterial trauma who had open surgical treatment and fasciotomy, performing fasciotomy early as compared to late was associated with improved outcomes including lower amputation risk and shorter length of stay. The favorable outcomes observed with early fasciotomy persisted in models adjusting for clinical covariates and across multiple clinical subgroups. Our findings provide evidence supporting a clinical benefit associated with early fasciotomy in lower extremity arterial trauma.

Our most significant finding is that patients in the Early Fasciotomy Group were significantly less likely to undergo limb amputation. In fact, early timing of fasciotomies was an independent predictor of limb salvage. A large series of fasciotomies in combat patients found similar results. Soldiers who underwent in-theater fasciotomies had 50\% lower amputation rates than those who had delayed fasciotomies (7). Others have shown that prophylactic fasciotomies (22) and fasciotomies performed at the time of vascular repair (14) were less likely to result in ECS (22), which is a known risk factor for amputation and limb morbidity. In addition, early fasciotomy was associated with decreased LOS. However, the longer LOS, in the Late Fasciotomy Group may be partially related to increased wound infection and amputation rates. Others have described increased hospital LOS in patients undergoing fasciotomies (22) and there is evidence that delayed therapeutic fasciotomy in the setting of ECS leads to significantly increased hospital LOS (8).

In our original analysis, iliac artery injury was more common in the Late Fasciotomy Group. Some authors consider iliac artery injury as a marker for abdominal vascular injury which can be associated with more severe trauma, especially when associated with bowel injury and pelvic fracture. Such an association with bowel injury, for example, could potentially explain higher wound infection rate and longer hospital stay in Late Fasciotomy Group. To address this issue, we performed an additional bivariate analysis after excluding iliac vascular injury from the study group. Our findings revealed significantly higher major limb amputation and longer total hospital stay in the Late Fasciotomy group and were thus similar to those found in the original analysis. In other secondary analysis, we included patients with surgical repair of a lower extremity arterial injury who did not have fasciotomy performed. Of note, the No Fasciotomy Group was significantly different in a number of parameters including fewer popliteal injuries and concomitant venous injuries and fractures and thus may represent a cohort at lower risk for ECS and adverse limb outcomes. We observed no difference in amputation rates between the early fasciotomy group compared to a combined group of patients with arterial injury and surgical repair who did not have an early fasciotomy (No Fasciotomy and Late Fasciotomy Groups). Thus, there is no evidence 
that performing an early fasciotomy in itself has associated risk for adverse limb outcomes. Taken together our findings are consistent with the possibility that appropriate use of early fasciotomy may improve limb outcomes in extremity arterial injury.

There were significantly fewer wound infections in patients who underwent early fasciotomy (6.6\% versus $14.5 \%)$. Similar findings were reported by others $(3,8)$. Higher wound infection may be related to muscle necrosis which can occur as a consequence of a delayed treatment of ECS (4). Wound infection has been described to occur in up to $25 \%$ of patients who undergo fasciotomy (5) and is cited by many as a reason to avoid fasciotomy. However, there is conflicting evidence as to whether wound infection is solely responsible for limb amputation $(8,16)$.

There were no demographic differences between the Early and Late Fasciotomy cohorts. This is similar to the series reported by Williams et al who found no difference in patients with vascular extremity trauma who had fasciotomies less than and more than 12 hours after their index procedure (2). Injury characteristics were different between the cohorts. A significantly higher proportion of patients in the Late Fasciotomy Group had iliac artery injury (23\% vs. $5.9 \%)$ and this may possibly reflect a decreased vigilance in performing fasciotomies in the setting of iliac artery trauma due to the expectation of multiple channels of collateral flow to the lower extremity. In one small series comparing patients with arterial extremity injuries who underwent prophylactic or no fasciotomies, there were 0 and $6 \%$ iliac injuries in the former and latter groups, respectively (22).

Although penetrating trauma was more common in this series there was no difference between the proportion of blunt and penetrating trauma between the Early and Late Fasciotomy groups. In one large series of patients with extremity trauma, mechanism of injury did not independently predict need for a fasciotomy (11). These observations suggests that mechanism of trauma, in of itself, unlikely influences the decision to perform fasciotomies.

Despite the fact that prolonged hypotension has been shown to be related to increased chance of fasciotomy (11) and limb loss (2), presenting SBP was no different between our 2 groups of patients. Likewise, other surrogates of injury severity including ISS and GCS were not different between groups. There was no difference in associated injuries (venous injury, nerve injury, fracture) between the groups despite the fact that presence of a combined arterial and venous injury is a relative indication for a prophylactic fasciotomy $(12,15)$. Finally, there was no difference between the groups based on the type of surgery (repair versus ligation) performed and this too contrasts with evidence that arterial ligation is associated with development of ECS (24). It is possible that arterial ligations performed were not of main vessels in the lower extremity, and may simply have been ligations of bleeding branches with intact in-line flow to the foot, thus explaining the contradiction with existing literature.

Our study has multiple limitations. First, the NTDB represents a "convenience sample" of data reported by participating facilities (23). Thus, we cannot describe incidence rates for the entire population. Second, the available coding did not describe whether the fasciotomy was performed during the index surgical procedure. In more than $90 \%$ of the cases the time interval between the two procedures was coded as 0 , thus it is highly likely that they were performed concurrently. Third, the compartment syndrome code is not specific to lower leg ECS and therefore thigh ECS may be included in the dataset. Also, because compartment syndrome was coded using both ICD-9 codes and a specific complication code it was not possible to precisely determine when, after initial presentation or in the post-operative period, it occurred: Due to this database shortcoming ECS was not used in the analysis. 
Fourth, due to retrospective nature of this study and limitation of the database, we were unable to ascertain whether the initial revascularization was successful and whether there was any delay in revascularization that may have influenced patient outcomes. Fifth, wound infection is not specific to the leg and may include abdominal wounds that may have been used to gain proximal control during intraoperative management of extremity trauma. Sixth, long-term follow up of these patients is not available. Similarly, due to inherent limitation of the database, we were unable to complete assessment regarding return to normal activities, employment, and other quality of life measures after limb salvage. However, the strengths of our sample include multicenter data collection, large size, and racial diversity. Our description is the largest available sample describing fasciotomy use and outcomes in lower extremity arterial trauma.

In summary, fasciotomies performed within 8 hours of arterial extremity injury treatment are associated with significantly improved limb outcomes and shortened hospital LOS when compared to fasciotomies performed at later time points. Our observational findings support further prospective studies to refine clinical recommendations regarding patient selection for prophylactic fasciotomy in the setting of lower extremity arterial injury.

\section{Acknowledgments}

Dr. Hamburg is supported by grants from the National Institutes of Health (HL083781 and HL102299).

\section{References}

1. MacGowan W. Acute ischaemia complicating limb trauma. J Bone Joint Surg Br. 1968; 50:472-81. [PubMed: 5726900]

2. Williams AB, Luchette FA, Papaconstantinou HT, Lim E, Hurst JM, Johannigman JA, et al. The effect of early versus late fasciotomy in the management of extremity trauma. Surgery. 1997; 122:861-6. [PubMed: 9347868]

3. Guerrero A, Gibson K, Kralovich KA, Pipinos I, Agnostopolous P, Carter Y, et al. Limb loss following lower extremity arterial trauma: what can be done proactively? Injury. 2002; 33:765-9. [PubMed: 12379385]

4. Finkelstein JA, Hunter GA, Hu RW. Lower limb compartment syndrome: course after delayed fasciotomy. J Trauma. 1996; 40:342-4. [PubMed: 8601846]

5. Heemskerk J, Kitslaar P. Acute compartment syndrome of the lower leg: retrospective study on prevalence, technique, and outcome of fasciotomies. World J Surg. 2003; 27:744-7. [PubMed: 12732999]

6. Kim JY, Buck DW, Forte AJ, Subramanian VS, Birman MV, Schierle CF, et al. Risk factors for compartment syndrome in traumatic brachial artery injuries: an institutional experience in 139 patients. J Trauma. 2009; 67:1339-44. [PubMed: 20009687]

7. Ritenour AE, Dorlac WC, Fang R, Woods T, Jenkins DH, Flaherty SF, et al. Complications after fasciotomy revision and delayed compartment release in combat patients. J Trauma. 2008; 64:S153S161. [PubMed: 18376159]

8. Sheridan GW, Matsen FA III. Fasciotomy in the treatment of the acute compartment syndrome. J Bone Joint Surg Am. 1976; 58:112-5. [PubMed: 1249096]

9. Wall CJ, Richardson MD, Lowe AJ, Brand C, Lynch J, de Steiger RN. Survey of management of acute, traumatic compartment syndrome of the leg in Australia. ANZ J Surg. 2007; 77:733-7. [PubMed: 17685947]

10. Austin OM, Redmond HP, Burke PE, Grace PA, Bouchier-Hayes DB. Vascular trauma--a review. J Am Coll Surg. 1995; 181:91-108. [PubMed: 7599783]

11. Branco BC, Inaba K, Barmparas G, Schnuriger B, Lustenberger T, Talving P, et al. Incidence and predictors for the need for fasciotomy after extremity trauma: A 10-year review in a mature level I trauma centre. Injury. 2010 Epub ahead of print. 
12. Cargile JS III, Hunt JL, Purdue GF. Acute trauma of the femoral artery and vein. J Trauma. 1992; 32:364-70. [PubMed: 1548726]

13. Jensen SL, Sandermann J. Compartment syndrome and fasciotomy in vascular surgery. A review of 57 cases. Eur J Vasc Endovasc Surg. 1997; 13:48-53. [PubMed: 9046914]

14. Fainzilber G, Roy-Shapira A, Wall MJ Jr, Mattox KL. Predictors of amputation for popliteal artery injuries. Am J Surg. 1995; 170:568-70. [PubMed: 7492002]

15. Abouezzi Z, Nassoura Z, Ivatury RR, Porter JM, Stahl WM. A critical reappraisal of indications for fasciotomy after extremity vascular trauma. Arch Surg. 1998; 133:547-51. [PubMed: 9605919]

16. Rush DS, Frame SB, Bell RM, Berg EE, Kerstein MD, Haynes JL. Does open fasciotomy contribute to morbidity and mortality after acute lower extremity ischemia and revascularization? J Vasc Surg. 1989; 10:343-50. [PubMed: 2778898]

17. Pyne D, Jawad AS, Padhiar N. Saphenous nerve injury after fasciotomy for compartment syndrome. Br J Sports Med. 2003; 37:541-2. [PubMed: 14665597]

18. Velmahos GC, Theodorou D, Demetriades D, Chan L, Berne TV, Asensio J, et al. Complications and nonclosure rates of fasciotomy for trauma and related risk factors. World J Surg. 1997; 21:247-52. [PubMed: 9015166]

19. Bermudez K, Knudson MM, Morabito D, Kessel O. Fasciotomy, chronic venous insufficiency, and the calf muscle pump. Arch Surg. 1998; 133:1356-61. [PubMed: 9865656]

20. Fitzgerald AM, Gaston P, Wilson Y, Quaba A, McQueen MM. Long-term sequelae of fasciotomy wounds. Br J Plast Surg. 2000; 53:690-3. [PubMed: 11090326]

21. Frink M, Klaus AK, Kuther G, Probst C, Gosling T, Kobbe P, et al. Long term results of compartment syndrome of the lower limb in polytraumatised patients. Injury. 2007; 38:607-13. [PubMed: 17306263]

22. Field CK, Senkowsky J, Hollier LH, Kvamme P, Saroyan RM, Rice JC, et al. Fasciotomy in vascular trauma: is it too much, too often? Am Surg. 1994; 60:409-11. [PubMed: 8198329]

23. National Trauma Data Bank (NTDB) Version 7.2 Dataset Manual. American College of Surgeons; 2010.

24. Mullins RJ, Lucas CE, Ledgerwood AM. The natural history following venous ligation for civilian injuries. J Trauma. 1980; 20:737-43. [PubMed: 7411662]

\section{Appendix 1. Inclusion and Exclusion Criteria (ICD-9 Description) Inclusion Criteria}

Injury to:

902.53 iliac artery

904.4 common femoral artery

904.1 superficial femoral artery

904.41 popliteal artery

904.51 anterior tibial artery

904.53 posterior tibial artery

Open Vascular Repair:

$38-38.09$ incision of vessels

38.08 incision of lower limb arteries

$38.3-38.39$ resection of vessel with anastomosis 
$38.4-38.49$ resection of vessel with replacement

$38.6-38.69$ other excision of vessel

38.8-38.89 other surgical occlusion of vessels

39.2 other shunt or vascular bypass

39.29 other (peripheral) vascular shunt or bypass

39.3 suture of vessel

39.3 suture of unspecified blood vessel

39.31 suture of artery

39.4 revision of vascular procedure

39.41 control of hemorrhage following vascular surgery

39.56 repair of blood vessel with tissue patch graft

39.57 repair of blood vessel with synthetic graft

39.58 repair of blood vessel with unspecified type of patch graft

39.59 other repair of vessel

\section{Additional Procedure:}

83.14 Fasciotomy

\section{Exclusion Criteria}

Injury to other blood vessels:

900 head and neck

901.xx thorax

903.xx upper extremity

902.xx abdomen (except 902.53)

\section{Additional injuries:}

$851-854$ intracranial injuries

952 spinal cord injuries

925 - 929 crushing injuries

940 - 949 burns

\section{Miscellaneous:}

Patients declared dead on arrival 


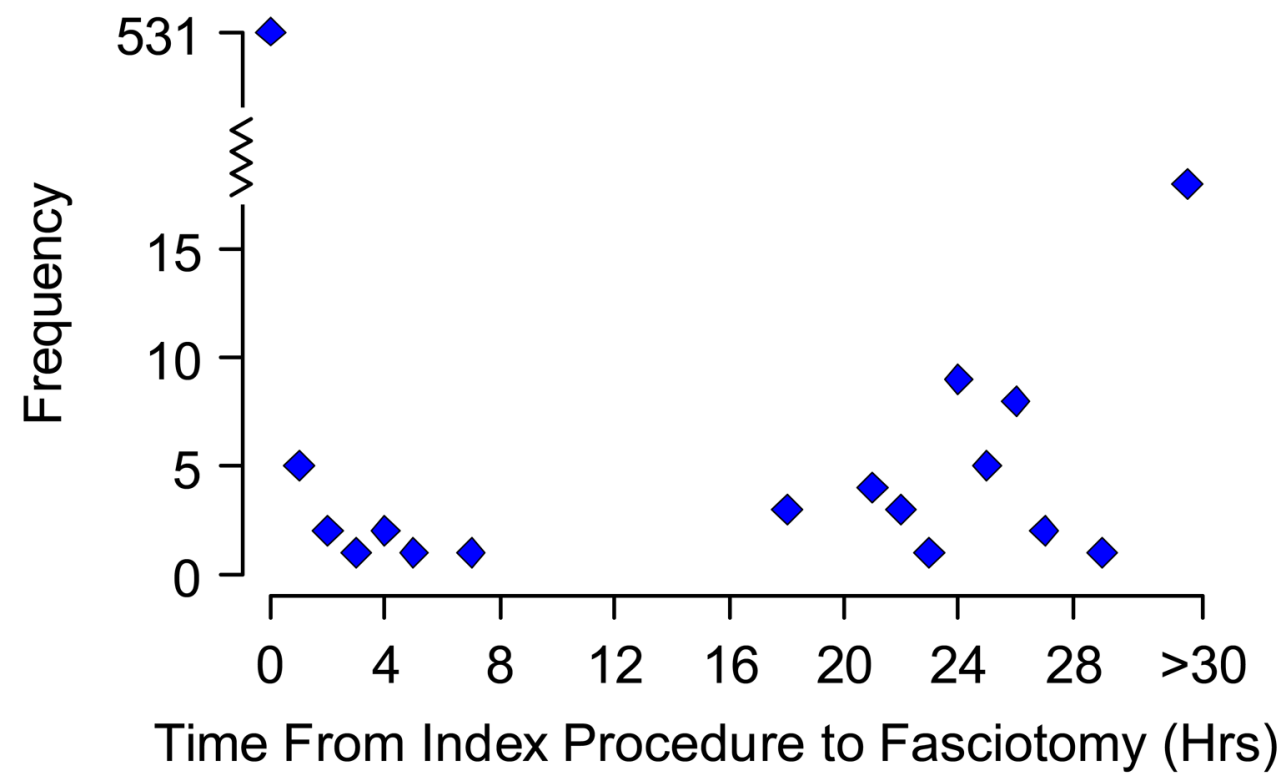

Figure 1.

The frequency of fasciotomies performed in the patient cohort over time. Thirty three late fasciotomy procedures were performed more than 30 hours after vascular repair. 


\section{Amputation in Early vs. Late Group}

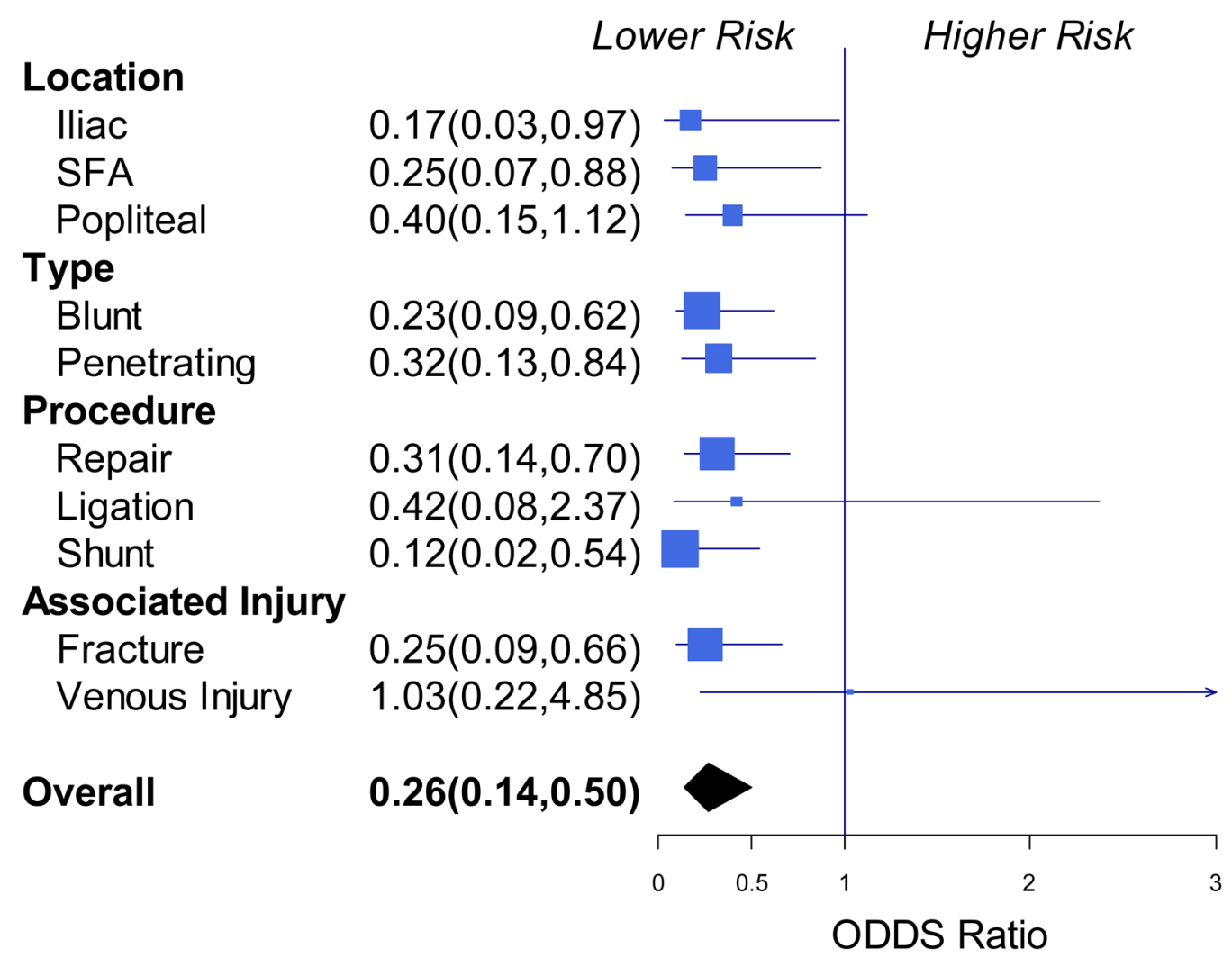

Figure 2.

Forrest plot of subgroup analysis of amputation rates between the Early and Late Fasciotomy Groups defined by location and mechanism of injury, type of repair, and presence of associated injury. 
Table 1

Demographic Characteristics of Early and Late Fasciotomy Groups

\begin{tabular}{|l|c|c|c|c|}
\hline Characteristic & Overall (N=612) & Early Fasciotomy (N=543) & Late Fasciotomy (N=69) & p-value \\
\hline Age & & & & \\
\hline Mean \pm SD & $31 \pm 11.2$ & $31 \pm 11.2$ & $30.8 \pm 11.1$ & 0.913 \\
\hline Median and IQ-Range & $27.5(22-37)$ & $28(22-37)$ & $27(22-36)$ & \\
\hline Gender, $N(\%)$ & & & & \\
\hline Male & $543(88.7 \%)$ & $483(89 \%)$ & $60(87 \%)$ & 0.685 \\
\hline Female & $69(11.3 \%)$ & $60(11 \%)$ & $9(13 \%)$ & \\
\hline Race, $N(\%)$ & & & & $16(24.6 \%)$ \\
\hline Caucasian & $172(29.3 \%)$ & $156(29.8 \%)$ & $30(46.2 \%)$ & 0.564 \\
\hline African American & $273(46.4 \%)$ & $243(46.5 \%)$ & $13(20 \%)$ & \\
\hline Hispanic & $107(18.2 \%)$ & $94(18 \%)$ & $6(9.2 \%)$ & \\
\hline Other & $36(6.1 \%)$ & $30(5.7 \%)$ & & $14(22.6 \%)$ \\
\hline Payment Type, $N(\%)$ & & & $48(77.4 \%)$ & 0.757 \\
\hline Self-pay & $144(25.2 \%)$ & $130(25.5 \%)$ & & \\
\hline Insurance & $428(74.8 \%)$ & $380(74.5 \%)$ & $19(28.8 \%)$ & 0.695 \\
\hline Hospital Region, $N(\%)$ & & & $19(28.8 \%)$ & \\
\hline Northeast & $193(33 \%)$ & $174(33.5 \%)$ & $121.2 \%)$ & \\
\hline South & $142(24.3 \%)$ & $123(23.7 \%)$ & & \\
\hline Midwest & $111(19 \%)$ & $97(18.7 \%)$ & & \\
\hline West & $139(23.8 \%)$ & $125(24.1 \%)$ & & \\
\hline
\end{tabular}

Note: due to missing fields total number of patients was less than 612 for certain characterisitics 
Table 2

Injury Type, Severity, and Associated Injuries in Early and Late Fasciotomy Groups

\begin{tabular}{|c|c|c|c|c|}
\hline Characteristic & Overall $(N=612)$ & Early Fasciotomy (N=543) & Late Fasciotomy (N=69) & p-value \\
\hline \multicolumn{5}{|l|}{ Injury Site, $N(\%)$} \\
\hline Iliac & $48(7.8 \%)$ & $32(5.9 \%)$ & $16(23.2 \%)$ & $<0.001$ \\
\hline Superficial Femoral & $202(33 \%)$ & $180(33.1 \%)$ & $22(31.9 \%)$ & \\
\hline Popliteal & $294(48 \%)$ & $265(48.8 \%)$ & $29(42 \%)$ & \\
\hline Tibial & $68(11.1 \%)$ & $66(12.2 \%)$ & $2(2.9 \%)$ & \\
\hline \multicolumn{5}{|l|}{ Injury Type, $N(\%)$} \\
\hline Blunt & $226(36.9 \%)$ & $201(37 \%)$ & $25(36.2 \%)$ & 0.999 \\
\hline Penetrating & $386(63.1 \%)$ & $342(63 \%)$ & $44(63.8 \%)$ & \\
\hline \multicolumn{5}{|c|}{ Injury Severity, Mean $\pm S D$} \\
\hline Presenting SBP, mmHg & $115.1 \pm 36.8$ & $115.2 \pm 37.4$ & $114.6 \pm 31.5$ & 0.899 \\
\hline Presenting GCS & $13.7 \pm 3.4$ & $13.7 \pm 3.4$ & $13.5 \pm 3.6$ & 0.682 \\
\hline Presenting ISS & $13.5 \pm 8$ & $13.4 \pm 8.3$ & $14.4 \pm 5.8$ & 0.334 \\
\hline \multicolumn{5}{|l|}{ Associated Injuries, $N(\%)$} \\
\hline Fracture & $287(46.9 \%)$ & $257(47.3 \%)$ & $30(43.5 \%)$ & 0.609 \\
\hline Nerve Injury & $61(10 \%)$ & $55(10.1 \%)$ & $6(8.7 \%)$ & 0.833 \\
\hline Venous Injury & $240(39.2 \%)$ & $219(40.3 \%)$ & $21(30.4 \%)$ & 0.118 \\
\hline \multicolumn{5}{|l|}{ Target Procedure, $N(\%)$} \\
\hline Repair & $384(62.7 \%)$ & $341(62.8 \%)$ & $43(62.3 \%)$ & 0.966 \\
\hline Ligation & $101(16.5 \%)$ & $89(16.4 \%)$ & $12(17.4 \%)$ & \\
\hline Temporary Shunt & $127(20.8 \%)$ & $113(20.8 \%)$ & $14(20.3 \%)$ & \\
\hline
\end{tabular}

SBP: systolic blood pressure, GCS: Glascow Coma Score, ISS: Injury severity score. 
Table 3

Complications and Outcomes in Early and Late Fasciotomy Groups

\begin{tabular}{|c|c|c|c|c|}
\hline Characteristic & Overall $(\mathrm{N}=612)$ & Early Fasciotomy (N=543) & Late Fasciotomy $(\mathrm{N}=69)$ & p-value \\
\hline \multicolumn{5}{|l|}{ Complications, $N(\%)$} \\
\hline DVT & $31(5.1 \%)$ & $27(5 \%)$ & $4(5.8 \%)$ & 0.769 \\
\hline $\mathrm{PE}$ & $4(0.7 \%)$ & $4(0.7 \%)$ & $0(0.0 \%)$ & 0.999 \\
\hline Wound Infection & $46(7.5 \%)$ & $36(6.6 \%)$ & $10(14.5 \%)$ & 0.028 \\
\hline \multicolumn{5}{|l|}{ Outcomes } \\
\hline Lower Limb Amputation, N (\%) & $63(10.3 \%)$ & $46(8.5 \%)$ & $17(24.6 \%)$ & $<0.001$ \\
\hline \multicolumn{5}{|l|}{ Total Length of Stay (days) } \\
\hline Mean \pm SD & $19.1 \pm 20.2$ & $18.5 \pm 20.7$ & $24.2 \pm 14.7$ & 0.007 \\
\hline Median and IQ-Range & $14(8-23)$ & $14(8-23)$ & $20(12-33)$ & \\
\hline \multicolumn{5}{|l|}{ ICU Length of Stay (days) } \\
\hline Mean \pm SD & $5.6 \pm 10$ & $5.3 \pm 10.2$ & $7.3 \pm 7.8$ & 0.294 \\
\hline Median and IQ-Range & $2(1-6)$ & $2(1-5)$ & $3(2-10)$ & \\
\hline In-hospital Mortality, N (\%) & $23(3.8 \%)$ & $21(3.9 \%)$ & $2(2.9 \%)$ & 0.999 \\
\hline
\end{tabular}

DVT; deep venous thrombosis, PE; pulmonary embolism, ICU; intensive care unit, IQ; interquartile 